\title{
Lebensqualität bei Patienten mit häuslicher Beatmung
}

\section{Quality of Life in Patients with Home Mechanical Ventilation}

Autoren

Institute
W. Windisch ${ }^{1}$, C. P. Criée ${ }^{2}$

Abteilung Pneumologie, Universitätsklinik Freiburg.

Ev. Krankenhaus Göttingen-Weende e.V., Abteilungen Pneumologie, Beatmungsmedizin und Schlaflabor, Bovenden-Lenglern.

\section{Bibliografie}

DOI $10.1055 / \mathrm{s}-2006-932152$

Pneumologie 2006; 60; 539-546

(C) Georg Thieme Verlag KG

Stuttgart · New York

ISSN 0934-8387

\section{Korrespondenzadresse}

PD Dr. med. Wolfram Windisch

Abteilung Pneumologie

Universitätsklinik Freiburg

Killianstraße 5

79106 Freiburg

windisch@med1.ukl.uni-frei-

burg.de

Serienherausgeber

D. Köhler, Schmallenberg

B. Schönhofer, Hannover

\section{Zusammenfassung}

Die gesundheitsbezogene Lebensqualität wird als mehrdimensionales psychologisches Konstrukt definiert, welches das subjektive Befinden eines Patienten auf physischer, psychischer, sozialer und funktionaler Ebene beschreibt. Die Evaluation der gesundheitsbezogenen Lebensqualität hat zunehmend an Bedeutung in der Bewertung medizinischer Therapiestrategien bei der Behandlung chronischer und nicht heilbarer Erkrankungen gewonnen und ist insbesondere auch bei $\mathrm{Pa}$ tienten mit häuslicher Beatmung und schweren objektiven Einschränkungen im Alltag von entschiedener Bedeutung. Moderne Instrumente, wie der Severe Respiratory Insufficiency (SRI) Questionnaire, sind speziell für diese Patientengruppe entwickelt worden und eignen sich zur Beurteilung des Therapieeffektes einer häuslichen Beatmung auf die gesundheitsbezogene Lebensqualität im Rahmen wissenschaftlicher Studien. Arbeiten der letzten Jahre haben gezeigt, dass die psychische Befindlichkeit bei stabiler Krankheitssituation nach Einleitung einer häuslichen Beatmung trotz erheblicher körperlicher Beeinträchtigungen normal sein kann und dass die gesundheitsbezogene Lebensqualität entscheidend von der Grunderkrankung abhängig ist. Dabei gilt der positive Effekt der häuslichen Beatmung auf die gesundheitsbezogene Lebensqualität bei Patienten mit thorakal-restriktiven und den meisten neuromuskulären Erkrankungen als bewiesen. Widersprüchlich sind jedoch die Daten zur gesundheitsbezogenen Lebensqualität bei COPD. Hier sind prospektive kontrollierte Studien mit Anwendung der modernen spezifischen Messinstrumente in der Zukunft nötig, um den Effekt der häuslichen Beatmung auf die gesundheitsbezogene Lebensqualität bei der COPD zuverlässig zu untersuchen.

\section{Abstract}

Health-related quality of life (HRQL) is defined as a psychological construct describing the subjectively experienced health status based on different components of health including physical state, psychological well-being, social relations and functional capacities. Assessment of HRQL has become steadily more essential in order to evaluate the costs and benefits of modern treatment modalities in patients with chronic and incurable diseases. This is particularly true for patients with home mechanical ventilation (HMV) and severe objective limitations in daily living. Modern instruments such as the Severe Respiratory Insufficiency (SRI) Questionnaire have been specifically designed for clinical trials which aim at assessing the effects of HMV on HRQL in these patients. Recent work has shown that mental health in clinically stable patients following establishment of HMV can be normal despite substantial physical handicaps. Further, HRQL has been shown to be predominantly influenced by the underlying disease. Accordingly, there is increasing evidence that HMV provides beneficial effects on HRQL in patients with neuromuscular and thoracic rib cage diseases, but in contrast, this remains still controversial in patients with COPD. Here, prospective controlled trials using modern specific instruments for HRQL assessment are required in the future to reliably evaluate the effects of HMV on HRQL in patients with COPD. 


\section{Einleitung}

Die Lebenserwartung hat sich insbesondere in den Industrienationen im letzten Jahrhundert stetig erhöht. So konnte zum Beispiel in den USA bedingt durch den zunehmenden medizinischen Therapieerfolg die Lebensspanne im 20. Jahrhundert um 30 Jahre verlängert werden [1]. Auch wenn der größte Anteil der hinzugewonnenen Lebenszeit in die erste Hälfte des letzten Jahrhunderts fällt, hat sich in den USA die Lebensspanne zwischen 1970 und 2000 noch um 6 Jahre verlängert, wovon zwischen 1990 und 2000 immerhin noch 1,5 Jahre Überlebenszeit gewonnen wurden, was insbesondere durch die zunehmend erfolgreiche Behandlung und Prävention von kardiovaskulären Erkrankungen zu erklären ist [1]. Allerdings wird bei vielen chronischen Erkrankungen eine Verbesserung des Langzeitüberlebens erreicht, ohne dass dabei das Grundleiden kuriert wird. Daher ist im Zuge der Spezialisierung der Medizin zunehmend die Frage entstanden, ob die dazu gewonnene Lebenszeit für den individuellen Patienten auch lebenswert ist $[2,3]$.

Diese Frage nach der Lebensqualität muss insbesondere bei Patienten mit chronischer Atempumpinsuffizienz gestellt werden, die bei weit fortgeschrittener Erkrankung auf eine häusliche Beatmungstherapie eingeleitet werden. Da es sich bei diesem Kollektiv meist um Patienten im Endstadium ihrer Erkrankung handelt, die nach objektiven Kriterien schwerste Einschränkungen im Alltagsleben mit sich bringt, ist die Frage nach dem Einfluss einer häuslichen Beatmung auf die Lebensqualität entscheidend für diese Patienten.

Die Lebensqualitätsforschung ist ein interdisziplinäres Feld, das Mediziner, Psychologen, Statistiker, Ökonomen und verschiedene Experten der Administration des Gesundheitswesens vereint [2]. Sie geht von einem weit gefassten biopsychosozialen Krankheitsmodell aus, bei dem auf dem Boden der subjektiven Einschätzung des Patienten innerpsychische Regulations- und Bewertungsprozesse evaluiert werden [2]. Da dieser Ansatz in erster Linie Aspekte der Gesundheit fokussiert, wird Lebensqualität in dieser Begriffsdefinition abgegrenzt von der Lebensqualität aus materieller, ökonomischer, sozialer, politischer und religiöser Perspektive. Man spricht daher von der gesundheitsbezogenen Lebensqualität (health-related quality of life) [2]. Diese Begriffsbezeichnung ist in der Literatur allerdings nicht einheitlich, was die Schwierigkeiten bei einer Begriffsdefinition untermauert $[2,4,5]$. Es besteht aber international zunehmend Einigkeit darüber, dass die gesundheitsbezogene Lebensqualität als mehrdimensionales psychologisches Konstrukt zu verstehen ist, welches das subjektive Befinden eines Patienten auf vier minimal zu definierenden Ebenen charakterisiert, nämlich unter Berücksichtigung physischer, psychischer, sozialer und funktionaler Aspekte [6]. Mit dieser patientenzentrierten Definition rückt die subjektive Patientenwahrnehmung in den Vordergrund. Objektive Kriterien oder Beurteilungen von „außen“ stehen im Hintergrund.

Die subjektive Wahrnehmung des Patienten wird nicht nur vom Charakter der Grunderkrankung, sondern auch entscheidend vom Verlauf derselben wie auch von der Behandlung beeinflusst. Daneben kann Lebensqualität abhängig von einer optimistischen bzw. pessimistischen Grundeinstellung deutlich unterschiedlich bewertet werden und von vorangegangenen Lebenserfahrungen, Erwartungen und persönlichen Einstellungen abhängig sein $[2,6]$.
Methodische Aspekte bei der Erfassung von Lebensqualität bei Patienten mit häuslicher Beatmung

\section{Messinstrumente}

Fragebogen dominieren bei der Erfassung der gesundheitsbezogenen Lebensqualität. Dadurch wird die subjektive Sichtweise des Patienten hinsichtlich seiner Einschränkungen unter methodischen Gesichtspunkten in den Mittelpunkt gerückt. Ein standardisiertes Interview kann alternativ eingesetzt werden. Eine Fremdbeurteilung von „außen“, z.B. durch ärztliches Personal oder Angehörige kann die Eigenbeurteilung ergänzen, diese aber nicht ersetzen.

Fragebogen eignen sich der guten Vergleichbarkeit wegen insbesondere für den Einsatz bei großen Patientenkollektiven im Rahmen wissenschaftlicher Studien. Einzelfallstudien können die Lebensqualität jedoch dann zielsicherer beschreiben, wenn individuelle, komplexe und ggf. seltene Faktoren nachvollzogen werden sollen, die durch einen Fragebogen nicht erfasst werden können. Als weitere Methoden werden Therapieabbruchraten und Selbstbeobachtungen (Tagesprotokoll) vorgeschlagen [2]. Viele Patienten finden ihre eigene und sehr persönliche Art und Weise, wie sie ihr subjektives Befinden zum Ausdruck bringen. Diese individuellen Ausdruckweisen sagen im Einzelfall eventuell mehr als die Ergebnisse von Fragebögen. So ist in $\mathbf{A b b} \mathbf{1}$ ist ein Gedicht eines Patienten mit häuslicher Beatmung bei amyotropher Lateralsklerose gezeigt.

Ein Fragebogen zur Messung der gesundheitsbezogenen Lebensqualität muss definierten Anforderungen und Test-Gütekriterien genügen. Der Nachweis solcher psychometrischer Kennwerte ist daher unerlässlich [2,3,7]. In diesem Sinne muss der Fragebogen objektiv und damit unabhängig vom Untersucher sein. Dies betrifft die Erhebung, Auswertung und Interpretation der Daten. Der Fragebogen muss zudem über ein hohes Maß an Zuverläs-

\section{Therapie}

\section{Einem Rosenstrauß lauwarmes Wasser geben und Zucker ins Glas ist wiekünstliche Atmung. Er hält sich dabei länger.}

\author{
Rudolf Jüdes 1994, ALS, \#eimbeatmung
}

Abb. 1 Tanka = fünfzeilige Form der reimlosen japanischen Dichtung eines Patienten mit amyotropher Lateralsklerose (ALS) und häuslicher Beatmung. Ein Tanka besteht aus einem Oberstollen mit 5:7:5 Silben und einem Unterstollen mit 7:7 Silben. Der Autor bemerkt, dass die festgelegten 31 Silben in 2 Atemzügen gesprochen werden müssen und dass die strenge, meditative Form des Silbenmaßes Atemdisziplin erfordert. 


\begin{tabular}{|c|c|c|c|c|}
\hline Fragebogen & Abkürzung & Literatur & Skalen & Zielgruppe \\
\hline $\begin{array}{l}\text { MOS 36-Item Short-Form Health } \\
\text { Survey }\end{array}$ & SF-36 & {$[11,12,13,14]$} & 8 & krankheitsübergreifend \\
\hline Sickness Impact Profile & SIP & {$[9,10]$} & 12 & krankheitsübergreifend \\
\hline Nottingham Health Profile & NHP & [8] & 6 & krankheitsübergreifend \\
\hline $\begin{array}{l}\text { Hospital Anxiety and Depression } \\
\text { Scale }\end{array}$ & HAD & {$[21]$} & 2 & krankheitsübergreifend \\
\hline $\begin{array}{l}\text { Chronic Respiratory Disease } \\
\text { Questionnaire }\end{array}$ & CRQ & [22] & 4 & COPD \\
\hline $\begin{array}{l}\text { St. George's Respiratory } \\
\text { Questionnaire }\end{array}$ & SGRQ & [23] & 3 & COPD \\
\hline $\begin{array}{l}\text { Fragebogen zur chronischen } \\
\text { Heimbeatmung }\end{array}$ & $\mathrm{FCH}$ & [24] & 5 & häusliche Beatmung \\
\hline Dresdner Fragebogen & & [40] & & $\begin{array}{l}\text { häusliche Beatmung im } \\
\text { Kindesalter }\end{array}$ \\
\hline $\begin{array}{l}\text { Maugeri Foundation Respiratory } \\
\text { Failure item set }\end{array}$ & MRF-28 & {$[25]$} & 3 & $\begin{array}{l}\text { chronische respiratorische } \\
\text { Insuffizienz }\end{array}$ \\
\hline $\begin{array}{l}\text { Severe Respiratory Insufficiency } \\
\text { Questionnaire }\end{array}$ & SRI & {$[26,27,28]$} & 7 & häusliche Beatmung \\
\hline
\end{tabular}

Tab. 1 Krankheitsübergreifende und krankheitsspezifische Fragebogen zur Erfassung der gesundheitsbezogenen Lebensqualität, die bei Patienten mit häuslicher Beatmung eingesetzt wurden sigkeit (Reliabilität) verfügen, also einen hohen Grad an Genauigkeit besitzen, mit dem die Lebensqualität gemessen wird. In diesem Sinne muss das Testergebnis gut reproduzierbar sein. Wichtigstes Gütekriterium bildet die Gültigkeit (Validität). Diese gibt an, ob der Sachverhalt, den es zu messen gilt, tatsächlich mit dem entsprechenden Instrument gemessen werden kann. So muss für einen Fragebogen, der vorgibt, die Lebensqualität zu messen, nachgewiesen sein, dass dieser Fragebogen tatsächlich die Lebensqualität und nicht irgendetwas anderes misst.

\section{Krankheitsübergreifende versus krankheitsspezifische Fragebögen}

Fragebögen zur Erfassung der Lebensqualität können krankheitsübergreifend (krankheitsunspezifisch) oder krankheitsspezifisch sein $[2,6]$. Erstere sind historisch älter und erlauben einen Vergleich zwischen verschiedenen spezifischen Gruppen. Wenn Untersuchungsergebnisse an Normalpersonen vorliegen, können sie darüber hinaus den Vergleich mit Normkollektiven erlauben. Die Universalität des Messinstrumentes wird mit seiner fehlenden Spezifität erkauft. Das bedeutet, dass bei spezifischen Fragestellungen krankheitsübergreifende Fragebogen nur unzureichend die Lebensqualität erfassen können. Darüber hinaus verfügen sie über eine eingeschränkte Änderungssensibilität. So können Veränderungen der Lebensqualität durch eine medizinische Intervention nur unscharf abgebildet werden, wenn ausschließlich krankheitsübergreifende Fragebogen eingesetzt werden.

Im Gegensatz dazu stehen krankheitsspezifische Instrumente, die Aspekte der gesundheitsbezogenen Lebensqualität bei einer definierten Erkrankung erfassen und daher in der Regel unerlässlich sind, wenn therapiebedingte Veränderungen der Lebensqualität möglichst genau abgebildet werden sollen. Der Nachteil krankheitsspezifischer Messinstrumente liegt entsprechend in der fehlenden Universalität, denn je spezifischer ein Messinstrument ist, desto kleiner ist sein Einsatzbereich. In der wissenschaftlichen Praxis wird zur Messung der gesundheitsbezogenen Lebensqualität eines definierten Patientenkollektivs der Einsatz sowohl eines spezifischen als auch eines unspezifischen Messinstrumentes empfohlen, um die Vorteile der Universalität und Vergleichbarkeit einerseits sowie der Spezifität und Änderungssensibilität andererseits zu vereinen. Die Fragebögen, die bei Patienten mit häuslicher Beatmung im Rahmen von Studien eingesetzt wurden, sind in Tab. 1 aufgeführt.

\section{Krankheitsübergreifende Fragebogen}

$\mathrm{Zu}$ den ersten gut validierten und häufig eingesetzten Fragebogen gehören das Nottingham Health Profile (NHP) [8] mit 28 Fragen in 6 Subskalen sowie das Sickness Impact Profile (SIP) $[9,10]$. Letzteres ist mit 136 Fragen in 12 Bereichen der gesundheitsbezogenen Lebensqualität eines der umfangreichsten Instrumente überhaupt. Übersetzungen liegen in mehreren Sprachen vor.

Das MOS 36-Item Short-Form Health Survey (SF-36) [11 - 14] enthält 36 Fragen in 8 Bereichen der subjektiven Gesundheit. Er ist gegenwärtig der wohl bekannteste und am meisten eingesetzte krankheitsübergreifende Fragebogen, da er kürzer als die meisten anderen Fragebogen ist, hervorragend validiert ist und darüber hinaus einen internationalen Vergleich mit Normkollektiven und anderen Patienten-Gruppen erlaubt. Seine internationale Verfügbarkeit basiert auf der Arbeit der International Quality of Life Assessment Group (IQOLA) [15-20].

Die Hospital Anxiety and Depression Scale (HAD-Skala) wurde als psychologisches Sreening-Instrument zur Erfassung von Angst und Depression bei Patienten internistischer Kliniken entwickelt [21]. Dabei zeigt die Skala (14 Fragen) keine Interferenz mit somatischen Symptomen von Angst und Depression, so dass physische Einschränkungen wie z. B. Schlafstörungen die Ergebnisse nicht verfälschen.

\section{Krankheitsspezifische Fragebogen}

Für den angloamerikanischen Sprachraum wurden der Chronic Respiratory Disease Questionnaire (CRQ) mit 4 Ebenen (Dyspnoe, Müdigkeit, emotionale Funktionen, Lebensbewältigung) [22] sowie der St. George's Respiratory Questionnaire (SGRQ) mit 3 Bereichen (Symptome, Aktivität, psychische Beeinträchtigungen) [23] speziell für die COPD entwickelt. Allerdings wurden beide Fragebogen an COPD-Patienten mit sehr unterschiedlichen Schweregraden ihrer Erkrankung entwickelt, so dass zu hinterfragen bleibt, ob diese Fragebogen für Patienten mit COPD und schwerer respiratorischer Insuffizienz geeignet sind. Aus diesem Grund wurden in letzten Jahren Fragebogen speziell für Patienten mit schwerer respiratorischer Insuffizienz entwickelt. Der erste war der Fragebogen zur chronischen Heimbeatmung (FCH) mit 57 Fragen [24]. Neben psychischen, sozialen 


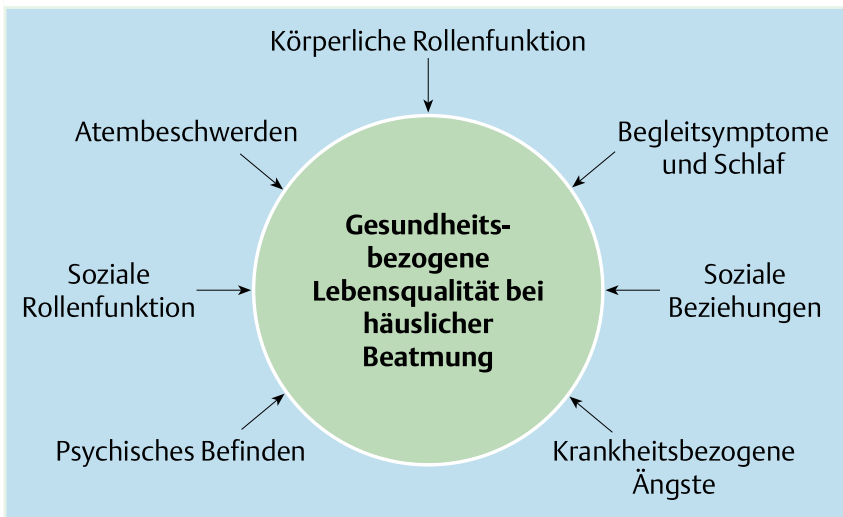

Abb. 2 Messmodel des Fragebogens zur Befindlichkeit bei Schwerer Respiratorischer Insuffizienz (SRI) mit sieben Ebenen der gesundheitsbezogenen Lebensqualität.

und allgemein funktionalen Aspekten der Lebensqualität konzentriert sich der Fragebogen auf funktionale Aspekte unter Beatmung und auf Nebenwirkungen der häuslichen Beatmung. Außerdem wurde das Maugeri Foundation Respiratory item set (MRF-28) für Patienten mit chronischer respiratorischer Insuffizienz entwickelt [25]. Dieser Fragebogen wurde im Rahmen einer Studie unter Einschluss von Patienten mit häuslicher Beatmung aber auch mit Sauerstofflangzeittherapie entworfen, wobei neben neuen Fragen auch solche aus älteren gut validierten Fragebogen (u.a. SGRQ, CRQ, SIP) mit aufgenommen wurden. Der Fragebogen enthält 28 Fragen (items) in drei Bereichen: Alltagsaktivität, kognitive Funktionsfähigkeit und Invalidität. Der MRF-28 verfügt über eine hohe inhaltliche Validität. Nachteilig sind jedoch eine geringe Varianzaufklärung bei der Konstruktvalidität infolge Faktorenanalyse sowie die Tatsache, dass der Fragebogen vor allem an COPD-Patienten validiert wurde. Der Einsatz bei Patienten mit restriktiven Erkrankungen oder neuromuskulären Erkrankungen ist daher nicht unproblematisch.

Auf dem Boden der Erfahrungen mit dem FCH wurde der Severe Respiratory Insufficiency (SRI) Questionnaire mit 49 Fragen in 7 Subskalen (Atembeschwerden, körperliche Rollenfunktion, Begleitsymptome/Schlaf, soziale Beziehungen, krankheitsbezogene Ängste, psychische Befindlichkeit und soziale Rollenfunktion) in deutscher Sprache entwickelt ( Abb. 2) [26]. Im Rahmen einer multizentrischen Validierungsstudie unter Einschluss von 226 Patienten mit chronischer respiratorischer Insuffizienz unterschiedlicher Genese und häuslicher Beatmungstherapie wurden für den SRI hohe psychometrische Kennwerte hinsichtlich Reliabilität und Validität nachgewiesen. Alle Fragen werden auf einer 5-Punkte-Likert-Skala mit Antwortmöglichkeiten zwischen 1 und 5 durch Ankreuzen beantwortet. Für jede Subskala und für die Summenskala wird ein Score zwischen 0 und 100 errechnet, wobei hohe Werte eine höhere Lebensqualität im Sinne des Konstruktes der Skala und tiefe Werte entsprechend eine niedrigere Lebensqualität anzeigen. Eine weitere Validierungsstudie konnte zeigen, dass der SRI über hohe psychometrische Kennwerte verfügt, wenn im speziellen COPD-Patienten zu ihrer Lebensqualität befragt werden, so dass der SRI insbesondere zur Erfassung der Lebensqualität von COPD-Patienten, die auf eine häusliche Beatmungstherapie eingeleitet werden, geeignet ist [27].

Es gibt außerdem zunehmend internationale Bemühungen, den SRI in verschiedene andere Sprachen zu übersetzen. Die Ergebnisse einer Validierungsstudie einer spanischen Übersetzung sind kürzlich publiziert worden [28]. Darüber hinaus werden eine holländische und eine englische Version des SRI in klinischen Studien getestet. Schließlich wird der SRI gegenwärtig ins Französische übersetzt.

\section{Studien zur Lebensqualität bei häuslicher Beatmung}

\section{Vergleich von Patienten mit häuslicher Beatmung und Normkollektiven}

Die schwedische Studie von Pehrsson u. Mitarb. aus dem Jahre 1994 war die erste Arbeit, die sich intensiv mit der Lebensqualität bei 39 Patienten mit einer restriktiven Ventilationsstörung und häuslicher Beatmung beschäftigt hat [29]. Zusammenfassend konnte aus der Vielzahl der Ergebnisse dieser Studie gemessen an den Werten einer gesunden Referenzgruppe eine hohe gesundheitsbezogene Lebensqualität insbesondere für den psychosozialen Bereich abgeleitet werden. Zwar zeigten sich im SIP vermehrte Einschränkungen im Vergleich zu Gesunden, verglichen mit anderen historischen Studienergebnissen, z. B. bei Patienten mit chronischen Rückenschmerzen oder rheumatoider Arthritis, waren diese Einschränkungen jedoch geringer ausgeprägt. Insgesamt lief die Beatmung mit nur wenigen Problemen, die meisten Patienten waren mit der Beatmungstherapie deutlich zufrieden. Die Schlafqualität wurde insgesamt als gut bezeichnet. Auch die Auswertung der HAD-Skala konnte keine erhöhte Prävalenz von Angst und Depression aufzeigen.

Diese guten Ergebnisse müssen vor dem Hintergrund einer Selektion des Patientenkollektivs betrachtet werden. Die mittlere Dauer der häuslichen Beatmung von 50 Monaten zeigt, dass in erster Linie Patienten mit hoher Compliance eingeschlossen wurden. Zum anderen war die tägliche Beatmungszeit mit durchschnittlich 7,7 Stunden trotz der langen Therapie-Dauer eher niedrig. Der tendenziell gute Allgemeinzustand der Patienten kommt auch dadurch zum Ausdruck, dass alle Befragten in der Lage waren, die insgesamt ca. 250 Fragen zu bearbeiten. Für viele andere Patienten könnte dies eine nicht überwindbare Mühe bedeuten. Schließlich wurden keine Patienten mit COPD in die Studie eingeschlossen.

In der großen Studie von Simonds u. Mitarb. wurden 105 von 180 eingeschlossenen Patienten mit häuslicher Beatmung mittels SF-36 untersucht [30]. Zwar war die gesundheitsbezogene Lebensqualität deutlich eingeschränkt im Vergleich zu einem Normalkollektiv. Diese Einschränkungen waren jedoch nicht wesentlich deutlicher als bei Patienten mit anderen chronischen Erkrankungen, die nicht auf eine häusliche Beatmung angewiesen waren.

Die größte Querschnittsstudie zur Lebensqualität bei häuslicher Beatmung umfasst insgesamt 226 Patienten mit unterschiedlichen Indikationen zur häuslichen Beatmung, bei denen der SF-36 eingesetzt worden war [31]. Dabei wurde in den Summenskalen deutlich, dass die körperliche Gesundheit im Vergleich zu einem Normalkollektiv und einem Patientenkollektiv mit chronischen Lungenerkrankungen deutlich eingeschränkt ist, was aufgrund der offensichtlichen und oftmals gravierenden körperlichen Behinderungen der Patienten zu erwarten ist. Allerdings zeigt sich keine richtungweisende Einschränkung in der psychischen Summenskala. Dies geht auch aus der Analyse der Subskalen hervor ( Abb. 3). Daraus ergibt sich, dass sogar schwere körperliche Beeinträchtigungen, wie sie bei Patienten mit häuslicher Beatmung vorliegen, nicht notwendigerweise zu psychischen Beeinträchtigungen führen müssen. 


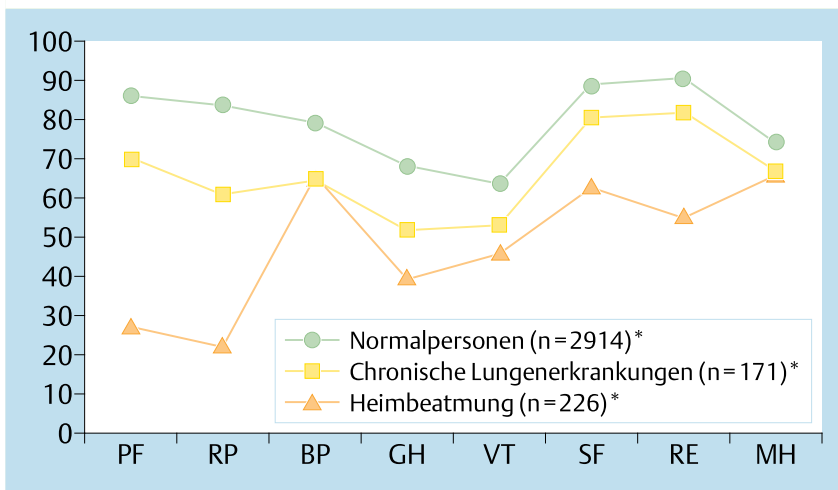

Abb. 3 Acht Subskalen des SF-36 bei Patienten mit häuslicher Beatmung im Vergleich mit einem historischen Normalkollektiv und einem historischen Kollektiv mit chronischen Lungenerkrankungen, die nicht abhängig von einem Respirator sind, modifiziert nach der Studie von Windisch u. Mitarb. [31]. Skalen von 0 ( = niedrigste Lebensqualität) bis 100 ( = höchste Lebensqualität). ${ }^{*}$ Referenz [14], ${ }^{* *}$ Referenz [31]

$\mathrm{PF}=$ Physical Functioning, $\mathrm{RP}=$ Role-Physical, $\mathrm{BP}=$ Bodily Pain, $\mathrm{GH}=$ General Health, VT = Vitality, SF = Social Functioning, RE = Role-Emotional, $\mathrm{MH}=$ Mental Health.

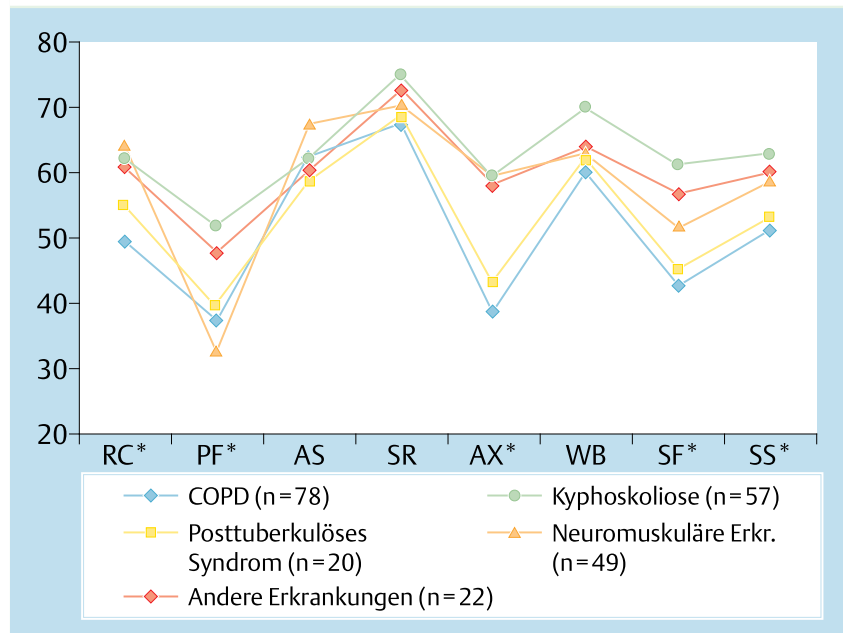

Abb. 4 Sieben Subskalen und eine Summenskala des SRI (Severe Respiratory Insufficiency Questionnaire) bei Patienten mit häuslicher Beatmung im Krankheitsgruppenvergleich, modifiziert nach der Studie von Windisch u. Mitarb. [26]. Skalen von 0 (= niedrigste Lebensqualität) bis 100 ( = höchste Lebensqualität).

$\mathrm{RC}=$ Respiratory Complaints, $\mathrm{PF}=$ Physical Functioning, $\mathrm{AS}=$ Attendant Symptoms and Sleep, SR = Social Relationships, AX = Anxiety, WB = Psychological Well-Being, $S F=$ Social Functioning, $S S=$ Summary Scale. ${ }^{*} P<0,05$.

Zusammenfassend bestehen somit deutliche Einschränkungen der subjektiv beschriebenen Lebensqualität bei Patienten mit häuslicher Beatmung im Vergleich zu Gesunden. Diese Einschränkungen sind jedoch nicht notwendigerweise größer als bei anderen Patienten, die nicht von einem Beatmungsgerät abhängig sind. Darüber hinaus betreffen diese Einschränkungen in erster Linie die körperliche Ebene. Dies führt nicht zwangsläufig, wie häufig geglaubt, auch zu psychischen Veränderungen, da das psychische Befinden bei vielen Patienten mit häuslicher Beatmung trotz schwerer körperlicher Beeinträchtigungen normal sein kann.
Einfluss der Grunderkrankung auf die Lebensqualität

Bei Patienten mit thorakal-restriktiven Erkrankungen und häuslicher Beatmung konnte in der Studie von Pehrsson u. Mitarb. gezeigt werden, das Patienten mit posttuberkulösem Syndrom eine schlechtere Lebensqualität hatten als Patienten mit anderen thorakal bedingten Restriktionen [29]. Dies zeigte sich auch in der großen Validierungsstudie des SRI [26]. Interessanterweise zeigt die Studie von Dellborg u. Mitarb. sehr ähnliche Unterschiede bei einem gemischten Patienten-Kollektiv mit chronischem Hypoventilations-Syndrom auf dem Boden thorakal-restriktiver Erkrankungen, die noch nicht auf eine häusliche Beatmung eingeleitet worden sind [32]. Dabei hatten Patienten mit posttuberkulösem Syndrom ebenfalls eine schlechtere gesundheitsbezogene Lebensqualität als Patienten mit Torsionsskoliose oder Post-Polio-Syndrom. Allerdings ist die Studie durch ihre eher geringe Fallzahl $(n=44)$, durch die heterogene Anzahl von Patienten in den einzelnen Krankheitsgruppen sowie durch die Tatsache limitiert, dass die Patientengruppen nicht für Alter und Geschlecht kontrolliert werden konnten, zumal ein fortgeschrittenes Alter als negativer Vorhersagewert vor allen Dingen für die körperliche Gesundheit angesehen werden darf [31]. Dennoch zeigen die Daten, dass krankheitsspezifische Unterschiede in der gesundheitsbezogenen Lebensqualität unabhängig davon bestehen können, ob eine häusliche Beatmungstherapie eingeleitet worden ist.

In der Studie von Simonds u. Mitarb. konnte anhand des SF-36 für Patienten mit COPD insgesamt eine schlechtere Lebensqualität nachgewiesen werden im Vergleich zu Patienten mit thorakal-restriktiven Erkrankungen [30]. Auf der funktionalen Ebene waren neuromuskulär Erkrankte am stärksten limitiert, was darin zu sehen ist, dass diese Patienten neben den funktionellen Einschränkungen durch die Atemstörung natürlich auch durch die generalisierte Muskellähmung funktionell limitiert sind. Allerdings sind in dieser Studie die einzelnen Subskalen des SF-36 in den verschiedenen Patienten-Gruppen nicht weiter quantifiziert, so dass eine weitere Analyse in dieser Studie nicht möglich ist.

In einer anderen Studie ( $\mathrm{n}=67)$, in der der FCH [24], die HADSkala [21] sowie eine modifizierte Version des Asthma Quality of Life Questionnaire (AQLQ) [33] eingesetzt wurde, konnte gezeigt werden, dass COPD-Patienten im Gegensatz zu SkoliosePatienten und neuromuskulär Erkrankten in der retrospektiven Befragung im Mittel keine Verbesserung ihrer gesundheitsbezogenen Lebensqualität infolge der häuslichen Beatmung angegeben haben [24]. Im Vergleich zu der Arbeit von Pehrsson u. Mitarb. [29] dokumentierten sich insgesamt deutlich höhere Auffälligkeiten für Angst und Depression, was, wie oben beschrieben, am ehesten in der Auswahl des Patientenkollektivs begründet ist.

In der bis dato größten Querschnittsstudie konnte sowohl anhand des SF-36 [31] als auch anhand des SRI [26] bestätigt werden, dass die gesundheitsbezogene Lebensqualität in starkem Maße abhängig von der Grunderkrankung ist, wobei für Patienten mit Kyphoskoliose die beste und für Patienten mit COPD die schlechteste Lebensqualität nachgewiesen worden ist ( Abb.4). Am stärksten sind die Unterschiede in den psychischen Bereichen der Lebensqualität. Hier dokumentieren sich die deutlichsten Unterschiede auf der Ebene der krankheitsbezogenen Ängste ( Abb.4), wobei neben den Patienten mit COPD auch solche mit einem posttuberkulösem Syndrom sehr viel deutlicher Ängste hatten als die anderen Patientengruppen. Dies deckt sich gut mit den Ergebnissen, die mittels HAD-Skala 
erarbeitet wurden, wo COPD-Patienten deutlich häufiger Ängste und Depressionen hatten als Patienten mit Kyphoskoliose oder neuromuskulären Erkrankungen [24].

Patienten mit Tracheotomie sind in der Regel stärker beeinträchtigt als solche, die über einen nichtinvasiven Beatmungszugang ventiliert werden. Dies betrifft insbesondere das Sprechen, das Essen, die Abhängigkeit von pflegenden Personen, den Komfort, Probleme, die sich aus dem künstlichen Beatmungszugang ergeben können, sowie die Tatsache, dass auch im Falle der Beatmungs-Pause häufig nicht über die natürlichen Atemwege geatmet werden kann [34]. Gelegentlich kann der sichere Beatmungszugang jedoch auch mehr subjektive Sicherheit und Vertrauen für den Patienten bedeuten.

Zusammenfassend hat die Grunderkrankung somit den stärksten Einfluss auf die gesundheitsbezogene Lebensqualität. Dies trifft auch für Patienten mit chronischer respiratorischer Insuffizienz zu, die einen Intensivstationsaufenthalt mit Langzeitbeatmung überlebt haben, so dass das Grundleiden als wesentlicher Faktor für die Lebensqualität im Langzeitverlauf angesehen werden kann [35]. Abgesehen vom Alter können objektive Parameter wie Daten der Lungenfunktion die gesundheitsbezogene Lebensqualität nur wenig voraussagen. Am stärksten scheinen noch die erhöhten Bikarbonat-Werte mit Einschränkungen der gesundheitsbezogenen Lebensqualität zu korrelieren. Dies betrifft sowohl Patienten mit chronischer ventilatorischer Insuffizienz, die auf eine häusliche Beatmung eingeleitet worden sind [29], als auch solche, die noch nicht beatmet werden [32]. Dabei konnte interessanterweise gezeigt werden, dass Verbesserungen der Lebensqualität gemessen mit dem SRI abhängig von der Bikarbonat-Elimination infolge suffizienter Beatmung waren [36]. Die schlechtere Lebensqualität bei COPD-Patienten kann darin begründet liegen, dass diese nicht nur durch eine ventilatorische Insuffizienz, sondern auch in erheblichem Maße durch eine pulmonale Insuffizienz mit Bronchospasmus, chronisch-produktivem Husten, Hypoxämie und den Folgen eines Cor pulmonale beeinträchtigt sind. Darüber hinaus kommt es bei COPD-Patienten häufiger zu Infektexazerbationen, von denen gezeigt wurde, dass sie in der Folge die gesundheitsbezogene Lebensqualität erheblich beeinträchtigen können $[37,38,39]$.

\section{Lebensqualität bei Kindern und Jugendlichen}

In allen oben erwähnten Studien wurden ausschließlich erwachsene Patienten eingeschlossen, so dass Rückschlüsse auf die Lebensqualität bei Kindern anhand dieser Studien nicht gezogen werden können. Daher wurde in einer Deutschland-weiten Multizenter-Studie die gesundheitsbezogene Lebensqualität bei häuslicher Beatmung im Kindes- und Jugendalter untersucht [40], wobei eigens für die Studie ein spezifischer Fragebogen entwickelt wurde. Zusammenfassend ergab sich danach eine hohe Patientenzufriedenheit, wobei interessanterweise eine zusätzlich durchgeführte Eltern-/Angehörigenbefragung diskrepante Ergebnisse bezüglich der gesundheitsbezogenen Lebensqualität erarbeiten konnte und zwar in dem Sinne, dass die Patienten ihre Lebensqualität höher einschätzten als die Eltern. Dies unterstreicht, dass eine Angehörigenbefragung die Patientenbefragung niemals ersetzen kann.

\section{Restriktive Erkrankungen}

Anhand retrospektiver Studien, Vergleich mit historischen Kollektiven und Erfahrungsberichten ist bereits frühzeitig klar geworden, dass die häusliche Beatmung bei dem größten Teil Patienten mit thorakal-restriktiven und neuromuskulären Erkran- kungen sowohl objektive Parameter wie den Gasaustausch oder Schlafqualität als auch subjektive Parameter wie Krankheitssymptome deutlich verbessern kann [41 - 45]. Auch eine Verlängerung des Lebens durch die häusliche Beatmung im Vergleich zur Sauerstofflangzeittherapie gilt als bewiesen [30,43,46,47]. Aus diesem Grund sind prospektive randomisierte Studien ethisch schwer zu rechtfertigen, weshalb die gesundheitsbezogene Lebensqualität in solchen Studien bei diesen Patienten nicht untersucht ist.

In einer nicht-kontrollierten Studie konnte gezeigt werden, dass bei Patienten mit thorakal-restriktiven und neuromuskulären Erkrankungen die Lebensqualität über einen Zeitraum von 18 Monaten verbessert werden konnte [48], wobei dies bei Patienten mit thorakal-restriktiven Erkrankungen deutlicher ist als bei Patienten mit neuromuskulären Erkrankungen [49]. Insbesondere bei Patienten mit thorakal-restriktiven Erkrankungen kann daher davon ausgegangen werden, dass trotz des Fehlens kontrollierter Studien nicht nur das Langzeitüberleben, sondern auch die gesundheitsbezogene Lebensqualität verbessert werden kann. So kommt es zu einer Verbesserung der körperlichen Belastbarkeit, der Symptome und der psychischen Befindlichkeit $[24,44]$. Zum Teil kann sogar eine Wiedereingliederung in den Beruf möglich sein [50]. Darüber hinaus konnte gezeigt werden, dass bei Patienten mit überwiegend restriktiven Ventilationsstörungen infolge häuslicher Beatmung nicht nur die gesundheitsbezogene Lebensqualität (SGRQ) verbessert wurde, sondern dass auch die mittels Pedometer gemessene Schrittzahl deutlich erhöht werden konnte [51]. Allerdings waren hier Lebensqualität und Schrittzahl nicht korreliert, so dass damit verdeutlicht ist, dass Fragebogen nicht alle Aspekte des Gesundheitsstatus abbilden können.

Retrospektiv verbesserte sich die Lebensqualität bei einer gemischten Gruppe mit neuromuskulären Erkrankungen vergleichbar mit Kyphoskoliose-Patienten [24]. Schwieriger ist jedoch die alleinige Beurteilung der Lebensqualität bei rasch progressiven neuromuskulären Erkrankungen wie bei der Muskeldystrophie Duchenne oder der amyotrophen Lateralsklerose. Bei Patienten mit Muskeldystrophie Duchenne konnte gezeigt werden, dass das Langzeitüberleben durch die häusliche Beatmung deutlich verbessert ist im Vergleich zu historischen Kollektiven und dass die Lebensqualität (SF-36), abgesehen von der körperlichen Rollenfunktion, nicht eingeschränkter ist als bei Patienten mit weniger stark progressiven Erkrankungen wie Kyphoskoliose, Post-Polio-Syndrom oder COPD [52]. Dabei wird die Lebenszufriedenheit dieser Patienten sehr häufig unterschätzt [53]. In der großen deutschen Querschnittsstudie konnte gezeigt werden, dass das psychische Befinden bei diesen Patienten sogar höher als in der Normpopulation war [31]. Aktuelle Daten konnte darüber hinaus darlegen, dass die gesundheitsbezogene Lebensqualität bei Duchenne-Patienten gemessen mit dem SF-36 im psychosozialen Bereich hoch ist und nicht schlechter ist bei Patienten mit fortgeschrittener chronischer ventilatorischer Insuffizienz und häuslicher Beatmungstherapie verglichen mit solchen Patienten, die bei besserer Lungenfunktion noch nicht beatmungspflichtig sind [54]. Aus diesem Grund kann davon ausgegangen werden, dass sich die Lebensqualität bei Duchenne-Patienten im Zuge der Entwicklung einer respiratorischen Insuffizienz mit der Notwendigkeit zur Einleitung einer häuslichen Beatmung nicht verschlechtert, sondern für den psychosozialen Bereich auf einem hohen Niveau gehalten werden kann. 
Bei Patienten mit amyotropher Lateralsklerose ergab eine kontrollierte Studie, dass die Verbesserungen der Lebenszufriedenheit durch die häusliche Beatmung nicht signifikant waren [55]. Neuere prospektiv erhobene Daten legen jedoch nahe, dass die häusliche Beatmung die Lebensqualität verbessern kann, wenn sie zur Behandlung schlafbezogener Atmungsstörungen eingesetzt wird [56]. Dabei ist die Lebensqualität in starkem Maße abhängig von der Atemmuskelschwäche [57]. In den beiden letzteren Studien wurde u.a. der SF-36, aber kein krankheitsspezifischer Fragebogen eingesetzt. Der Effekt der häuslichen Beatmung auf die Lebensqualität bei Patienten mit amyotropher Lateralsklerose ist daher nicht sicher zu beurteilen, solange spezifische Messinstrumente für diese besondere Erkrankung nicht entwickelt und prospektiv eingesetzt werden.

\section{COPD}

Gänzlich anderes ist die Situation bei der COPD. Während große kontrollierte Studien den Nutzen für die Akutexazerbation klar dargelegt haben $[43,58,59,60,61]$, ist bis dato ungeklärt, ob die Langzeitanwendung bei Patienten mit hyperkapnischer COPD einen Vorteil für die Patienten bringt. Nach den Ergebnissen der kontrollierten Studien führt die häusliche Beatmung bei der COPD zu keiner wesentlichen Verbesserung objektiver Funktionsparameter wie der Blutgasanalyse oder der Lungenfunktion und auch nicht des Langzeitüberlebens [61-68].

Die Studienergebnisse zum Einfluss der häuslichen Beatmung auf die gesundheitsbezogene Lebensqualität bei der COPD sind zum Teil unterschiedlich. In zwei Arbeiten konnten keine wesentlichen Verbesserungen durch die häusliche Beatmung dokumentiert werden $[69,70]$. In zwei weiteren Arbeiten zeigte sich eine signifikante Verbesserung der gesundheitsbezogenen Lebensqualität, wobei in diesen beiden Studien im Gegensatz zu den ersteren beiden u.a. der SGRQ eingesetzt wurde $[63,71]$. Fragebögen, die spezifisch die Probleme der Patienten mit häuslicher Beatmung erfragen, wurden jedoch in keiner dieser Studien eingesetzt. Im Gegensatz dazu wurde in der jüngsten Arbeit gezeigt, dass Verbesserungen der Lebensqualität bei diesen Patienten nur dann sicher abgebildet werden konnten, wenn ein für die schwere respiratorische Insuffizienz spezifischer Fragebogen (MRF-28) eingesetzt wurde [66]. Dies unterstreicht, wie wichtig der Einsatz spezifischer Instrumente ist, wenn Lebensqualität bei einer solchen spezifischen Patientengruppe erfasst werden soll.

In allen zitierten Studien zur COPD wurden allerdings nur sehr geringe Inspirationsdrücke eingesetzt [67], so dass der oftmals deutlich erhöhte Kohlendioxidpartialdruck im Sinne einer insuffizienten Ventilation nicht oder nur sehr wenig gesenkt werden konnte. Auf der andern Seite konnte in anderen Studien gezeigt werden, dass auch bei Patienten mit COPD und schwerer Hyperkapnie der Kohlendioxidpartialdruck im Sinne einer suffizienteren Ventilation deutlich gesenkt werden konnte [36, 72,73]. Für eine abschließende Beurteilung der Frage, ob die häusliche Beatmung die Lebensqualität bei Patienten mit stabiler hyperkapnischer COPD verbessern kann, sind somit Studien erforderlich, die auf der einen Seite eine möglichst effektive Ventilation der Patienten garantieren und auf der anderen Seite die modernen und spezifischen Messinstrumente einsetzen.

\section{Literaturverzeichnis}

1 Lenfant $C$. Shattuck lecture-clinical research to clinical practice - lost in translation? N Engl J Med 2003; 349: $868-874$

2 Petermann F, Krischke N, Deuchert M. Methodische Probleme bei der Erfassung der Lebensqualität.In: Petermann F, Bergmann KC (Hrsg). Lebensqualität und Asthma. Berlin, München: Quintessenz, 1994: $29-42$

3 Windisch W, Friedel K, Matthys $\mathrm{H}$ et al. Gesundheitsbezogene Lebensqualität bei Patienten mit Heimbeatmung. Pneumologie 2002; 10: $610-620$

4 Hoffman LG, Rouse MW, Brin BN. Quality of life: a review. Journal of the American Optometric Association 1995; 66: 281 - 289

5 Wood-Dauphinee S. Assessing quality of life in clinical research: from where have we come and where are we going? J Clin Epidemiol 1999; 52: $355-363$

6 Testa MA, Simonson DC. Assesment of quality-of-life outcomes. N Engl J Med 1996; 334 (13): $835-840$

7 Lienert G, Raats U. Testaufbau und Testanalyse. Weinheim: Verlags Union, 1998

8 Hunt SM, McKenna SP, McEwen J et al. The Nottingham Health Profile: subjective health status and medical consultations. Soc Sci Med 1981; 15: $221-229$

9 Bergner M, Bobbitt RA, Pollard WE et al. The sickness impact profile: validation of a health status measure. Medical Care 1976; 14: 57-67

10 Bergner M, Bobbitt RA, Carter WB et al. The Sickness Impact Profile: development and final revision of a health status measure. Medical Care 1981; 19: 787-805

11 Ware JE Jr, Sherbourne CD. The MOS 36-item short-form health survey (SF-36). I. Conceptual framework and item selection. Medical Care 1992; 30: $473-483$

12 Ware JE Jr, Kosinski M, Bayliss MS et al. Comparison of methods for the scoring and statistical analysis of SF-36 health profile and summary measures: summary of results from the Medical Outcomes Study. Medical Care 1995; 33 (4 Suppl): AS264-279

13 Ware JE Jr. The SF-36 Health Survey. In: Spilker B (Hrsg). Quality of Life and Pharmacoeconomics in Clinical Trials., 1996: 337-345

14 Bullinger M, Kirchberger I. SF-36 Fragebogen zum Gesundheitszustand - Handanweisung. 1998

15 Aaronson NK, Acquadro C, Alonso J et al. International Quality of Life Assessment (IQOLA) Project. Qual Life Res 1992; 1: 349- 351

16 Bullinger $M$. German translation and psychometric testing of the SF-36 Health Survey: preliminary results from the IQOLA Project. International Quality of Life Assessment. Soc Sci Med 1995; 41: 1359 1366

17 Wagner AK, Gandek B, Aaronson NK et al. Cross-cultural comparisons of the content of SF-36 translations across 10 countries: results from the IQOLA Project. International Quality of Life Assessment. J Clin Epidemiol 1998; 51: 925 - 932

18 Ware JE Jr, Kosinski M, Gandek B et al. The factor structure of the SF-36 Health Survey in 10 countries: results from the IQOLA Project. International Quality of Life Assessment. J Clin Epidemiol 1998; 51: 11591165

19 Ware JE Jr, Gandek B, Kosinski M et al. The equivalence of SF-36 summary health scores estimated using standard and country-specific algorithms in 10 countries: results from the IQOLA Project. International Quality of Life Assessment. J Clin Epidemiol 1998; 51: 1167-1170

20 Ware JE Jr, Gandek B. Overview of the SF-36 Health Survey and the International Quality of Life Assessment (IQOLA) Project. J Clin Epidemiol 1998; 51: $903-912$

21 Zigmond AS, Snaith RP. The hospital anxiety and depression scale. Acta Psychiatrica Scandinavica 1983; 67: 361 - 370

22 Guyatt GH, Berman LB, Townsend $M$ et al. A measure of quality of life for clinical trials in chronic lung disease. Thorax 1987; 42: 773-778

23 Jones PW, Quirk FH, Baveystock CM. The St George's Respiratory Questionnaire. Respir Med 1991; 85 (Suppl B): 25 - 31

24 Windisch W, Petermann F, Laier-Groeneveld G et al. Lebensqualität bei Heimbeatmung. Med Klin 1997; 92 (Suppl 1): 95-100

25 Carone M, Bertolotti G, Anchisi F et al. Analysis of factors that characterize health impairment in patients with chronic respiratory failure. Quality of Life in Chronic Respiratory Failure Group. Eur Respir J 1999; 13: $1293-1300$

26 Windisch W, Freidel $K$, Schucher B et al. The Severe Respiratory Insufficiency (SRI) Questionnaire: a specific measure of health-related quality of life in patients receiving home mechanical ventilation. J Clin Epidemiol 2003; 56: $752-759$ 
27 Windisch W, Budweiser S, Heinemann F et al. The Severe Respiratory Insufficiency (SRI) Questionnaire: a specific measure of health-related quality of life (HRQL) in patients with COPD receiving home mechanical ventilation (HMV). Eur Respir J 2005; 26 (Suppl.49): 85s

28 López-Campos JL, Martínez IF, Jiménez AL et al. Transcultural adaptation and validation of the SRI questionnaire in Spanish patients receiving home mechanical ventilation. Eur Respir J 2005; 26 (Suppl.49): $5 \mathrm{~s}$

29 Pehrsson K, Olofson J, Larsson S et al. Quality of life of patients treated by home mechanical ventilation due to restrictive ventilatory disorders. Respir Med 1994; 88: 21 - 26

30 Simonds AK, Elliott MW. Outcome of domiciliary nasal intermittent positive pressure ventilation in restrictive and obstructive disorders. Thorax 1995; 50: 604-609

31 Windisch W, Freidel K, Schucher B et al. Evaluation of health-related quality of life using the MOS 36-Item Short-Form Health Status Survey in patients receiving noninvasive positive pressure ventilation. Intensive Care Med 2003; 29: 615-621

32 Dellborg C, Olofson J, Midgren B et al. Quality of life in patients with chronic alveolar hypoventilation. Eur Respir J 2002; 19: $113-120$

33 Juniper EF, Guyatt GH, Epstein RS et al. Evaluation of impairment of health related quality of life in asthma: development of a questionnaire for use in clinical trials. Thorax 1992; 47: 76-83

34 Bach JR. A comparison of long-term ventilatory support alternatives from the perspective of the patient and care giver. Chest 1993; 104 : $1702-1706$

35 Euteneuer S, Windisch W, Suchi S et al. Health-related quality of life in patients with chronic respiratory failure after long-term mechanical ventilation.Respir Med 2006, in press

36 Windisch $W$, Dreher $S$, Storre J et al. Nocturnal non-invasive positive pressure ventilation: Physiological effects on spontaneous breathing. Respir Physiol Neurobiol 2006, in press

37 Hurel D, Loirat P, Saulnier F et al. Quality of life 6 months after intensive care: results of a prospective multicenter study using a generic health status scale and a satisfaction scale. Intensive Care Med 1997; 23: $331-337$

38 Seemungal TA, Donaldson GC, Paul EA et al. Effect of exacerbation on quality of life in patients with chronic obstructive pulmonary disease. Am J Respir Crit Care Med 1998; 157: 1418-1422

39 Ambrosino N, Bruletti G, Scala $V$ et al. Cognitive and perceived health status in patient with chronic obstructive pulmonary disease surviving acute on chronic respiratory failure: a controlled study. Intensive Care Med 2002; 28: 170-177

40 Paditz E, Zieger S, Bickhard J et al. Lebensqualität im Kindes-, Jugendund jungen Erwachsenenalter: unterschiedliche Sichtweisen von Eltern und Kindern. Somnologie 2000; 4: 13-19

41 Anonymous. Clinical indications for noninvasive positive pressure ventilation in chronic respiratory failure due to restrictive lung disease, COPD, and nocturnal hypoventilation - a consensus conference report. Chest 1999; 116: $521-534$

42 Schönhofer B, Köhler D. Effect of non-invasive mechanical ventilation on sleep and nocturnal ventilation in patients with chronic respiratory failure. Thorax 2000; 55: $308-313$

43 Mehta S, Hill NS. Noninvasive ventilation. Am J Respir Crit Care Med 2001; 163: $540-577$

44 Shneerson JM, Simonds AK. Noninvasive ventilation for chest wall and neuromuscular disorders. Eur Respir J 2002; 20: 480 - 487

45 Simonds AK. Home ventilation. Eur Respir J 2003; 47 (Suppl): 38s -46s

46 Leger P, Bedicam JM, Cornette A et al. Nasal intermittent positive pressure ventilation. Long-term follow-up in patients with severe chronic respiratory insufficiency. Chest 1994; 105: 100-105

47 Buyse $B$, Meersseman $W$, Demedts $M$. Treatment of chronic respiratory failure in kyphoscoliosis: oxygen or ventilation? Eur Respir J 2003; 22: $525-528$

48 Domenech-Clar RF, Nauffal-Manzur DF, Perpina-Tordera MF et al. Home mechanical ventilation for restrictive thoracic diseases: effects on patient quality-of-life and hospitalizations. Respir Med 2003; 97: $1320-1327$

49 Nauffal D, Domenech R, Martinez Garcia MA et al. Noninvasive positive pressure home ventilation in restrictive disorders: outcome and impact on health-related quality of life. Respir Med 2002; 96: 777 - 783

50 Sawicka EH, Loh L, Branthwaite MA. Domiciliary ventilatory support: an analysis of outcome. Thorax 1988; 43: $31-35$
51 Schönhofer B, Ardes P, Geibel $M$ et al. Evaluation of a movement detector to measure daily activity in patients with chronic lung disease. Eur Respir J 1997; 10: 2814 - 2819

52 Simonds AK, Muntoni F, Heather $S$ et al. Impact of nasal ventilation on survival in hypercapnic Duchenne muscular dystrophy. Thorax 1998; 53: $949-952$

53 Bach JR, Campagnolo DI, Hoeman S. Life satisfaction of individuals with Duchenne muscular dystrophy using long-term mechanical ventilatory support. Am J Phys Med Rehabil 1991; 70: 129 - 135

54 Kohler M, Clarenbach CF, Boni L. et al. Quality of life, physical disability, and respiratory impairment in duchenne muscular dystrophy. Am J Respir Crit Care Med 2005; 172: 1032 - 1036

55 Pinto AC, Evangelista T, Carvalho $M$ et al. Respiratory assistance with a non-invasive ventilator (Bipap) in MND/ALS patients: survival rates in a controlled trial. J Neurol Sci 1995; 129 (Suppl): 19-26

56 Lyall RA, Donaldson N, Polkey MI et al. Respiratory muscle strength and ventilatory failure in amyotrophic lateral sclerosis. Brain 2001; 124 : 2000- 2013

57 Bourke SC, Shaw PJ, Gibson GJ. Respiratory function vs sleep-disordered breathing as predictors of QOL in ALS. Neurology 2001; 57: 2040 2044

58 Brochard L, Mancebo J, Wysocki M et al. Noninvasive ventilation for acute exacerbations of chronic obstructive pulmonary disease. N Engl J Med 1995; 333: $817-822$

59 Plant PK, Owen JL, Elliott MW. Early use of non-invasive ventilation for acute exacerbations of chronic obstructive pulmonary disease on general respiratory wards: a multicentre randomised controlled trial. Lancet 2000; 355: 1931 - 1935

60 Lightowler JV, Wedzicha JA, Elliott MW et al. Non-invasive positive pressure ventilation to treat respiratory failure resulting from exacerbations of chronic obstructive pulmonary disease: Cochrane systematic review and meta-analysis. BMJ 2003; 326: 185

61 Hill NS. Noninvasive ventilation for chronic obstructive pulmonary disease. Respir Care 2004; 49: $72-89$

62 Strumpf DA, Millman RP, Carlisle CC et al. Nocturnal positive-pressure ventilation via nasal mask in patients with severe chronic obstructive pulmonary disease. Am Rev Respir Dis 1991; 144: 1234-1239

63 Meecham Jones DJ, Paul EA, Jones PW et al. Nasal pressure support ventilation plus oxygen compared with oxygen therapy alone in hypercapnic COPD. Am J Respir Crit Care Med 1995; 152: 538 - 544

64 Gay PC, Hubmayr RD, Stroetz RW. Efficacy of nocturnal nasal ventilation in stable, severe chronic obstructive pulmonary disease during a 3-month controlled trial. Mayo Clin Proc 1996; 71: 533 - 542

65 Casanova C, Celli BRF, Tost L et al. Long-term controlled trial of nocturnal nasal positive pressure ventilation in patients with severe COPD. Chest 2000; 118: 1582 - 1590

66 Clini E, Sturani C, Rossi A et al. The Italian multicentre study on noninvasive ventilation in chronic obstructive pulmonary disease patients. Eur Respir J 2002; 20: 529-538

67 Wijkstra PJ, Lacasse Y, Guyatt GH et al. A meta-analysis of nocturnal noninvasive positive pressure ventilation in patients with stable COPD. Chest 2003: $124: 337$ - 343

68 Wijkstra PJ. Non-invasive positive pressure ventilation (NIPPV) in stable patients with chronic obstructive pulmonary disease (COPD). Respir Med 2003; 97: 1086 - 1093

69 Elliott MW, Simonds AK, Carroll MP et al. Domiciliary nocturnal nasal intermittent positive pressure ventilation in hypercapnic respiratory failure due to chronic obstructive lung disease: effects on sleep and quality of life. Thorax 1992; 47: 342 - 348

70 Sivasothy P, Smith IE, Shneerson JM. Mask intermittent positive pressure ventilation in chronic hypercapnic respiratory failure due to chronic obstructive pulmonary disease. Eur Respir J 1998; 11: 34-40

71 Perrin C, El Far Y, Vandenbos F et al. Domiciliary nasal intermittent positive pressure ventilation in severe COPD: effects on lung function and quality of life. Eur Respir J 1997; 10: 2835-2839

72 Windisch W, Vogel $M$, Sorichter $S$ et al. Normocapnia during nIPPV in chronic hypercapnic COPD reduces subsequent spontaneous $\mathrm{PaCO}_{2}$. Respir Med 2002; 96: 572 - 579

73 Windisch W, Kostic S, Dreher M et al. Outcome of patients with stable COPD receiving controlled NPPV aimed at maximal reduction of Pa$\mathrm{CO}_{2}$. Chest 2005; $128: 657-662$ 